\title{
ModLock: A Manual Connector for Reconfigurable Modular Robots
}

\author{
Jay Davey, Jimmy Sastra, Matt Piccoli, and Mark Yim
}

\begin{abstract}
Connection mechanisms are critical to many modular reconfigurable systems. This paper introduces the ModLock manual connection system which is both easy and fast to attach/detach (requires seconds) as well as strong (failure at $2.2 k N$ tensile load). This low cost, low profile connection system has been demonstrated on a variety of robot configurations including legged walkers, flying quadrotors and wheeled robots.
\end{abstract}

\section{INTRODUCTION}

One of the key features of a modular reconfigurable robot is its ability to adapt its morphology to the task required. While the modular robotics community has mostly focused on this feature by furthering self-reconfiguration mechanisms to enable the robot to reconfigure itself [18], [10], [15], we believe there is also value in fast manual reconfiguration to make modular robots practical and useful in the field with humans present.

Modular robots are generally constructed out of modular robotic hardware that can be of either homogeneous or heterogeneous design. In homogeneous systems, modular robots are built from identical robotic building blocks. Heterogeneous systems are built from modular hardware consisting of a limited number of modular building pieces. In this paper, modules refers to the system's modular building pieces. Modules on a heterogeneous system can have several functions and could provide actuation, skeleton, wheels, control or power services, to name a few.

The ability to rapidly reconfigure modules of a modular robot is advantageous in situations where a task needs to be solved quickly, but some information about the environment or task is not known beforehand. Examples can be found in space exploration, or emergency response such as search and rescue operations. This has been illustrated in the ICRA Planetary Contingency Competition [16], which simulates an emergency situation requiring a robotic solution. Teams must pack a suitcase, of airline regulation size and weight, knowing that they will need to solve some unknown task requiring a robotic solution. Once they arrive at the competition, the specifics of the tasks are announced and teams may only use what has been packed in their suitcase. In this competition robots are put together on-the-fly to suit the task. Being able to quickly build the hardware solution is critical for teams to complete tasks optimally.

Another application for rapid morphological changes in robots includes conceptual design. Here the focus is usually not optimization but rather, showing the potential to work as a proof of concept device. Modular robots can be put together faster than building a traditional robot from raw materials and components. Fast assembly and low cost components allows

GRASP Lab and Department of Mechanical Engineering and Applied Mechanics, at University of Pennsylvania, email: jaydavey, jsastra, piccoli, yim@ seas.upenn.edu many configurations to be tested to find the viable ones. This hardware versatility means the robot can be used for several different tasks.

One of the issues in building rapidly reconfiguring systems is attaining strong, but temporary, bonds between modules. There is typically a trade-off between strength and ease of reconfiguration. The classic example is the popular Lego toy system. Lego is a heterogeneous reconfigurable modular building system, with pieces that little require little effort to assemble, but are subject to falling apart easily under large mechanical loads. In this work, we describe a connection mechanism we have coined the "ModLock". This mechanism is small, fast, strong and easy to operate. We have experimentally tested the strength of our connector under tension, torsion and bending loads and have implemented the ModLock on a modular robot system.

Several terms are used in this paper to describe different connection mechanisms. In this paper, a connector is gendered if one half of the connector exhibits only male parts and the matching connector holds only female parts. A nut and bolt is an example of male and female parts where two nuts cannot be coupled together and vice versa. Magnets are included in the gendered connector category where only North poles can connect to South poles. Well known Lego bricks contain male 'studs' which are pressed into a receptacle on the under side, or female side, of the brick. Lego bricks exhibit the gendered connection principle. Hermaphroditic connectors are defined as connections that contains both male and female parts on both sides. Typically, hermaphroditic connectors are derived from gendered connection principles. Genderless connectors are connectors that do not follow any male-female rule for connecting pieces together and cannot be seen to hold both male and female parts. Train carriage couplings are one example of genderless connections. For comparison, another way of connecting two system pieces is to use a bolted connection. For example, two plates containing holes can be riveted together. This connection is termed a female-male-female (FMF) connection because a single male piece is used to connect two female pieces together.

\section{A. Related Work}

Previous connection mechanisms for modular robots and robotic construction kits have generally made a trade-off between strength and the time it takes to connect and disconnect robotic parts. Compliant mechanisms such as those used in Lego pieces for Lego Mindstorms, Topobo [13] and Molecubes [19] can be connected in seconds, but they do not support large loads. Recently, Cubelets [3] have become commercially available and their robotic modules connect together using magnetic force. While it is easy to 
connect all of these these systems together, it also requires relatively little force for them to break apart. This is mainly because most of these systems have been designed as toys for children to manually reconfigure. On the other hand, several modular robot systems are connected using screws such as CKbot V1 [12], PolyBot G1 [17], Bioloid [4] and Vex [5]. These bonds are very strong, but it takes much longer to connect system components together.

Thor [2], is a modular robot whose genderless connector design was specifically inspired by the ICRA Planetary Contingency competition. It is a hermaphroditic connection that utilizes magnets to quickly snap system pieces together, but also features holes to allow modules to be connected together using screws for added strength if necessary. The connector can be bonded in eight orientations.

Yamor [8] uses Velcro ${ }^{\mathrm{TM}}$ for an instantaneous connection like magnets, but has the added benefit of no restriction on orientation with which the faces can be connected. It is however a relatively weak bond for modular robot applications.

Nilsson [11] built the DRAGON connector who's focus was on automatic connection. Its design emphasized misalignment tolerance during docking. It has been shown to support $70 \mathrm{~kg}$ of tension force and weighs $170 \mathrm{~g}$. A connection made with the DRAGON connector can be manually reconfigured using four pins to manually override the automatic SMA latching feature. It has not been designed to disconnect under axial loading.

There are also now dozens of different self-reconfigurable systems that have been built to date and have their own styles of automatic connections. Most of them however are generally not manually reconfigured without being electrically powered. These self-reconfigurable systems include ATRON [6], the M-TRAN III [9], Superbot [14], CONRO [1], and recent work from JHU [7].

\section{ModLock DESIGN}

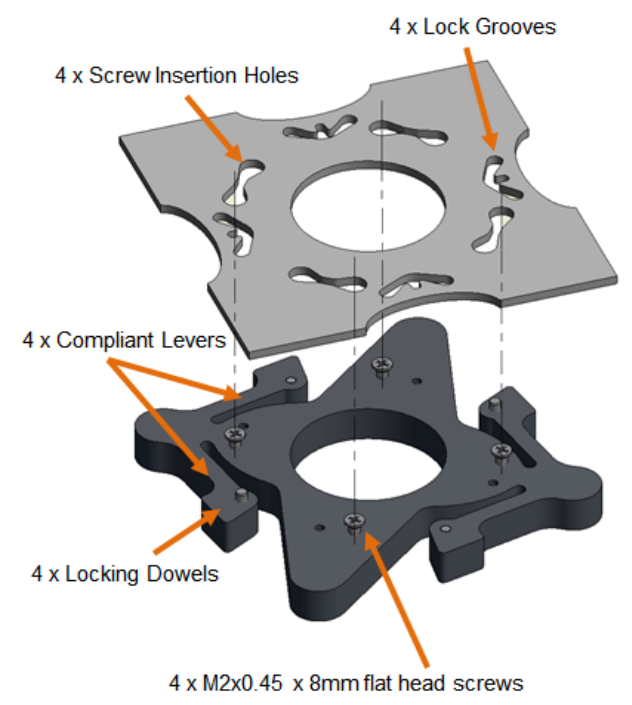

Fig. 1: The ModLock connector with the female connector (top) and compliant male connector plate assembly (bottom)

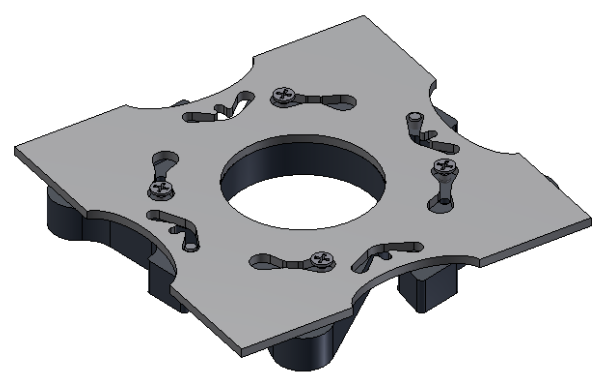

(a) Male connector plate assembly and female a pattern are pushed together

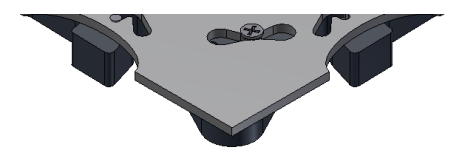

(b) Connectors twist to lock

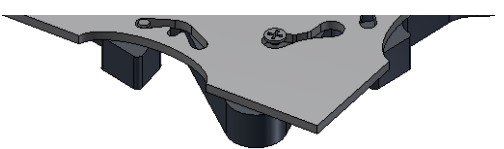

(c) Bi-directional unlock

Fig. 2: Method of connection

The ModLock mechanism utilizes a female-male-female connection principle. Female connectors are placed on all system modules. To connect two modules a male connector plate assembly is used to connect the two female patterns together. Effectively, it operates as two gendered connections where any system module holds a female connector and a male connector couples the female patterned modules together. Each male connector has four flat head screws embedded into the plate. The screws are not turned, rather the head geometry acts as hooks. To connect two modules together, the screws are aligned to fit into four custom shaped screw insertion holes, (Fig. 1). The plates are pressed together so the heads go through the screw insertion holes (Fig. 2a). The faces are twisted $12^{\circ}$ to align and tighten the male connector plate assembly with the female pattern. A stainless steel locking dowel on a compliant lever snaps into a lock groove to prevent the faces from untwisting. This is shown in Fig. 2b. Once the faces are tightened with respect to each other and the locking dowel locates in the lock groove, the connection between the male connector plate and the female connector is complete. A remaining female connector is secured to the other side of male connector plate to join two female connectors together.
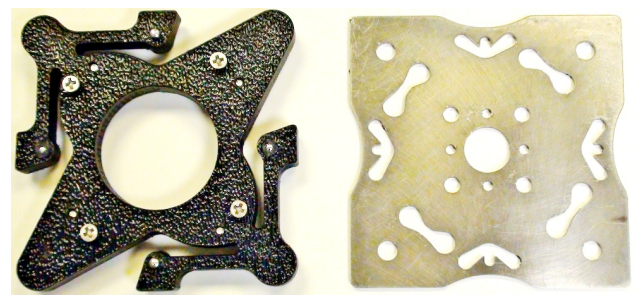

Fig. 3: Fabricated male connector plate assembly (left) and female connector pattern (right) 
To disconnect modules a user pushes on opposing compliant levers to disengage the locking dowels. He or she then twists the male connector plate assembly in either direction to disengage the screw heads. Modules can then be effortlessly pulled apart. Patterned features in the female connector are typically laser cut steel plates. The male connector plate body is $6 \mathrm{~mm}$ Acrylonitrile Butadiene Styrene (ABS) which includes the compliant lever. A male connector plate's compliant levers are pushed with a minimum of $40 \mathrm{~N}$ of force to unlock the locking dowel pin. This value was obtained using CAD software and built in Finite Element Analysis simulation (Fig. 4).

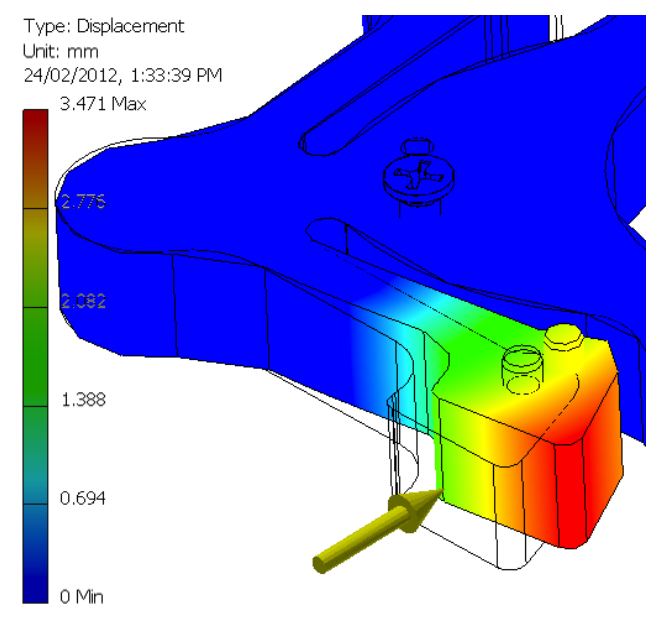

Fig. 4: Complaint Lever (ABS) with Locking Dowel Pin

In an earlier version (ModLock V1), the connector design was hermaphroditic (Fig. 5). Here the screw head and insertion holes are the same, however a locking pin, locking groove and compliant lever were integrated into the plate. When two plates were pressed together a pan head screw head would snap into one of the round hole features in the neighboring connection plate. To disconnect, the user would disengage the pan head screw by pushing on the lever on which it was attached. A single connection has fewer parts (no separate male plate) but it requires that all connector locations on modules contains both male and female parts. Each side of the connector was much more complicated containing five screws, four screw insertion holes, four locking grooves and a compliant lever. In our current version V3, shown fabricated in (Fig. 3), male elements are only required when modules are to be connected together. This reduces the total part complexity of a multimodule system.

One disadvantage of the V1 system is more difficult recovery from failures. If one side cannot undo its connector, or if a compliant lever gets jammed for any reason, neither side can disconnect. In the V3 version, both sides can disconnect from the male connector, increasing connector robustness.

ModLock V1 connected by twisting modules together only in the clockwise direction. This can be a problem for modules that are to be connected in a closed chain. The final module added to a closed chain makes two connections at once, but it cannot be made as one of the plates must twist in a counter-clockwise manner. ModLock V2 (Fig. 5) included the female-male-female mechanism but similarly can only be connected in one direction of twist. To fix the 'closing-theloop' problem, the ModLock V3 design allows for modules to connect and disconnect in either direction.

Moving the compliant lever arm and screw heads to a male part allows the female part to be much simpler and also made out of much stronger material like sheet steel. We've fabricated a pilot run of 250 pieces with the V3 female connector pattern, cut from 16 gage 316 stainless steel. 150 male connector plate assembly pieces have been cut and assembled from $6 \mathrm{~mm}$ ABS plastic, with $4 \mathrm{x} M 2.5 \times 0.45$ $\mathrm{x} 8 \mathrm{~mm}$ flat head Phillips screws and four $2 \mathrm{~mm}$ diameter $\mathrm{x}$ $8 \mathrm{~mm}$ long stainless steel dowel pins. The female connector pattern has also been placed on passive modules such as wheels and casters, showing how easily ModLock can be implemented.

A male connector plate assembly measures $60 \mathrm{~mm}$ on each side. The minimum size the matching female connector can be is also a $60 \mathrm{~mm}$ sided square.
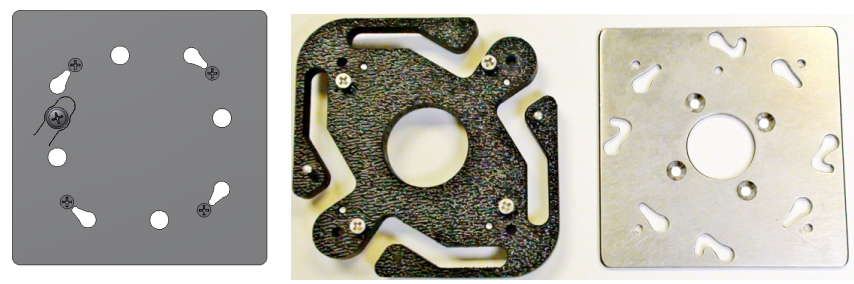

Fig. 5: Early hermaphroditic style connector (V1) (left). ModLock with single direction lock and unlock (V2) (right).

\section{EXPERIMENTS}

\section{A. Strength tests}

We conducted force experiments to find the maximum strength of our connector. We used an Instron tensile test bench (model \#4206) to experimentally determine the force vs displacement curve of the ModLock V3 connector and a Cooper Instruments load cell (model \#LFS 260 -50) to measure torque. Pairs of folded sheet metal frames laser cut with ModLock patterns were connected with the male connector plate and loaded until failure. The output of loading in pure tension is shown in Figure 7. We also conducted bending moment and pure torque tests to experimentally determine how many modules we can hold in a cantilever configuration.

\section{B. Results}

Two pairs of test connectors failed at around $2.2 k \mathrm{~N}$ of force in the tensile direction. The mode of failure was the M2 screws stripping out of the ABS male connector plate. Significant permanent plastic deformation occurred to the stainless steel brackets which suggests the connector should be used well below the ultimate strength if it is to remain a reusable connector. We have not yet determined what strength the connector should be rated at to ensure plastic deformation does not occur. The $2.2 k N$ load suggests the ModLock connector might be stronger in the tensile direction than the DRAGON Connector which held $0.69 k N$ [11] and 

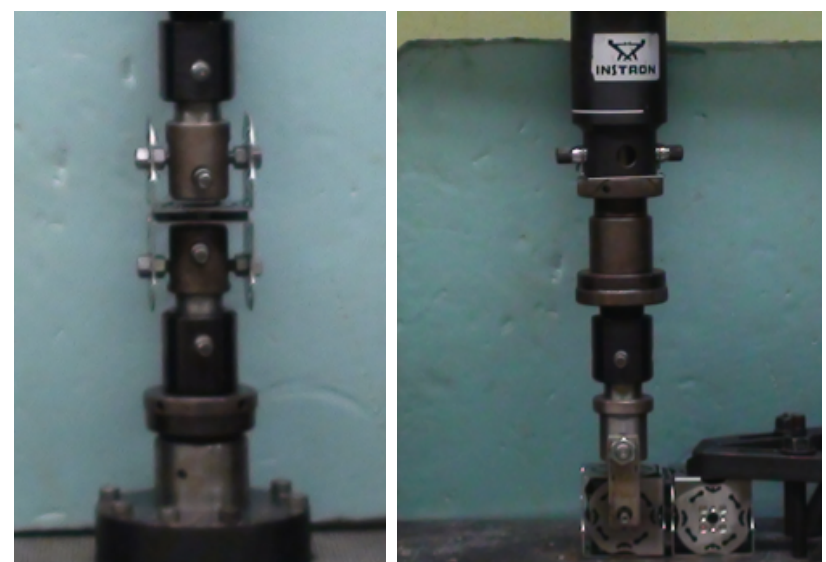

Fig. 6: Tensile (left) and bend (right) testing of the femalemale-female ModLock element with Instron 4206

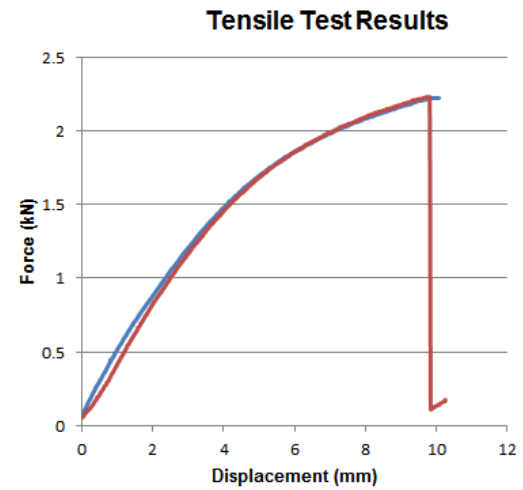

Fig. 7: Plot of two runs of tensile testing

was specifically designed to be heavy duty. The uncertainty comes in that the yield point of the ModLock V3 was not explicitly determined.

Our connector weighs in at $80 \mathrm{~g}$ for two female stainless steel patterns and a male connector plate. This gives us a strength to weight ratio of $27.5 \mathrm{kN} / \mathrm{kg}$ for tensile strength. By comparison, the DRAGON connector has a strength to weight ratio of $4.1 \mathrm{kN} / \mathrm{kg}$, but most of the weight in this connector is from the automated docking mechanism.

We tested two pairs of specimens under a bending load and they failed at $38 \mathrm{Nm}$ and $41 \mathrm{Nm}$ respectively. Again, significant plastic deformation occurred in the stainless steel brackets before the M2 screws eventually stripped out of the ABS connector plate. As a case study, we chose to implement the ModLock on CKBot reconfigurable robots. With the CKBot system a single U-Bar module weighs $0.320 \mathrm{~kg}$ and a single connector plate weighs $0.016 \mathrm{~kg}$. Its Center of Mass is $47 \mathrm{~mm}$ and $3 \mathrm{~mm}$. The overall dimensions are $90 \mathrm{~mm}$ and $6 \mathrm{~mm}$ respectively. Each combined module and connector piece pair that is attached will then have a combined $d_{\text {com }}=0.044 \mathrm{~m}, W=0.236 \mathrm{~N}$, and $l=0.096 \mathrm{~m}$, so that we can calculate the resulting torque $\tau=W d_{\text {com }}+$ $\sum_{i=1}^{n} t_{i} W\left(l+d_{\text {com }}\right)(i-1)$ with $n$ being the number of modules. At $n=16$ modules, the torque is $39 \mathrm{Nm}$, we have reached the measured strength under bending.

A pair of modules was tested under a pure torque loading.
A significant angle between the modules occurred at $15 \mathrm{Nm}$ and ultimate failure occurred at $17 \mathrm{Nm}$. The failure mode in this loading was the shear force from the locking pins expanding their press fit holes. Note that once the pins failed, the angle between the modules became $12^{\circ}$ and the M2 screws held the torque at higher values, but the connector would no longer able to support tensile loads.

Mechanical backlash of the connectors was tested by cantilevering six modules from a fixed seventh module, which applied torques between $12 \mathrm{Nmm}$ and $432 \mathrm{Nmm}$ on the connectors. The angles between the modules' adjacent faces were measured via image processing. This method accounted for any deformation in the module frame, giving values for the backlash in the connector only. An average backlash angle of $0.52^{\circ}$ with a standard deviation of $0.25^{\circ}$ and a correlation of 0.43 between angle and torque. The high standard deviation and low correlation suggest the backlash is a function of the M2 screw height, which was manually calibrated with relatively low precision. Assuming the same amount of backlash at the characteristic strength of 16 modules, this results in a positional error of $24 \mathrm{~mm}$ in $\mathrm{X}$, and $240 \mathrm{~mm}$ in $\mathrm{Z}$.

\section{Speed of Assembly}

Table I shows how long it takes to assemble a 5 module snake using the ModLock connector compared to CKBot V1 and Bioloid. CKBot V1 requires 4 socket head machine screws per face to connect two modules together. Each face contains four threaded holes. For the bioloid measurements we used the FR07-H101K hinge and FR07-S101K side frame set. We assume the servo horns have already been installed. Assembly then requires 1 hinge, 1 side frame, 4 nuts and 14 bolts between two servos. It is shown that the ModLock system is an order of magnitude faster to assemble and disassemble than using machine screws with nuts to join modular system pieces together. A novice user with a few minutes of practice on each system was tested in each case. Experienced users in all cases would be faster.

\section{TABLE I}

\begin{tabular}{|l|l|l|} 
Connector & $\begin{array}{l}\text { Assembly } \\
\text { Time (s) }\end{array}$ & $\begin{array}{l}\text { Disassembly } \\
\text { Time (s) }\end{array}$ \\
\hline CKBot with ModLock & 28 & 13 \\
CKBot V1 & 243 & 162 \\
Bioloid RX-28 & 1917 & 420
\end{tabular}

\section{IMPLEMENTATION}

\section{A. Modular Robotics}

The ModLock connector has been implemented on the new (unpublished) version of CKBot, a modular robot designed at the ModLab of the University of Pennsylvania. A ModLock mechanism is designed into each of the faces allowing any module to be connected in four different orientations on any face. A U-Bar (UB) module has $180^{\circ}$ of freedom, whereas the Continuous Rotation (CR) module provides unlimited turns like a motor. Modules are controlled and powered by a Control and Power Module (CM). 
A lightweight version of the ModLock connector was developed for use on flying vehicles. The compliant portion of the connector is identical to the standard connector, but is only $1.6 \mathrm{~mm}$ thick. These connectors were built into the design of a laser cut ABS reinforced foam core frame. A lightweight CR module with three ModLock faces was also built. The lightweight CR modules used high-torque, brushless, DC motors and custom controllers. A sensor was added to the motors, allowing for bi-directional rotation and low speed operation. Four to eight of these lightweight CR modules were attached to the frame in various configurations including a quadrotor, an octocopter, and a wheeled vehicle. A working quadrotor with four lightweight CR modules is pictured in Fig. 10b. We did not implement a ModLock connector on the output of the lightweight CR modules due to difficulties in mounting the connector to a propeller. The lightweight connectors experienced no failures from the $>5 \mathrm{~N}$ tension forces and $>100 \mathrm{Nmm}$ of axial torque; however, the locking dowel pins fatigued their press fit hole in the thinner ABS and fell out after a small number of connect-disconnect cycles.

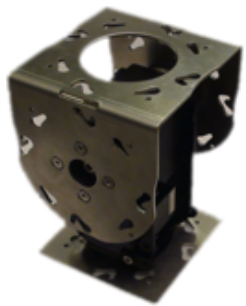

(a) U-Bar

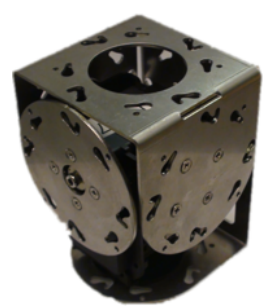

(b) $\mathrm{CR}$

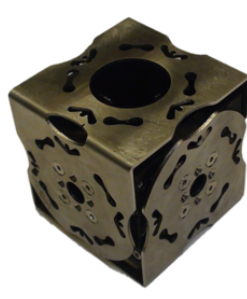

(c) Control or Power
Fig. 8: CKBot Modules with ModLock



(a) Hexapad (HP), Linear Actuator (LA), and Angled Framing (UF)
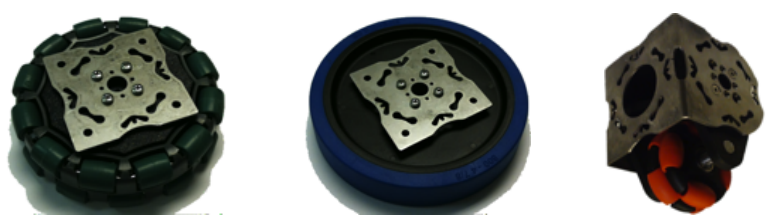

(b) Omni wheel, 4" wheel, and omni caster wheel modules

Fig. 9: CKBot passive attachments fitted with ModLocks

\section{B. Backward Compatibility}

Bi-bender male connector plates (Fig. 11) allows V2 and V3 ModLock female patterns to be connected to each other. However, whenever a robot is constructed and it requires modules in a closed loop, the V3 female ModLock must be used at the location where the loop will be closed. To unlock the loop, a V3 connector must be released first.



(a) Hexapods built from CKBot modules fitted with the ModLock

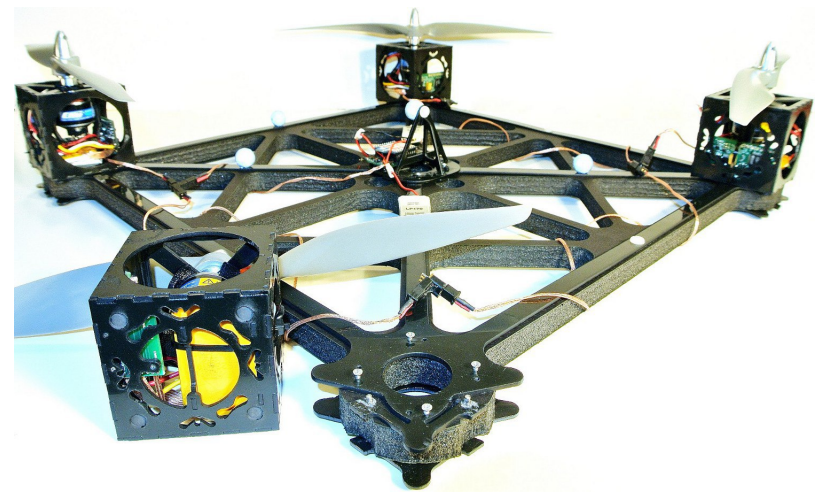

(b) Modular quadrotor with one disconnected lightweight CR module

Fig. 10: Configurations
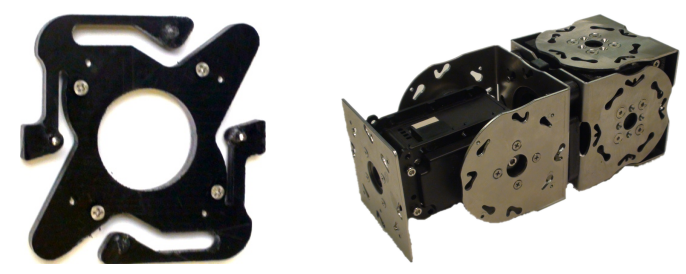

Fig. 11: Bi-bender male connector plate and application

\section{Quick Change End Effector}

ModLock is also in use on the Willow Garage PR2. In this case, the ModLock is used to quickly change end effectors, making the end effectors heterogeneous modules. A modified male ModLock connector is attached to the robot's wrist, while modified female connectors are attached to various end effectors. The PR2's custom wrist, seen in Fig. 12a, contains additional five gaps on the outer perimeter and an electrical brush block. The brush block provides power and communication to end effectors such as Willow Garage's gripper or a jamming gripper.

Guides enabled the PR2 to achieve the precision necessary to use the ModLock and allow for self-reconfiguration of end effectors. Engaging and disengaging of the ModLock is achieved by descending through the layers of the guides. Fig. 12b displays each uniquely keyed layer of the guide with arrows showing the motions allowed by each layer. Fig. 12a shows two end effectors located in the guides. Changing endeffectors can still be done manually and effortlessly by an operator of the PR2 without using the guides.

Fig. $12 \mathrm{~b}$ shows an exploded view of each of the layers 

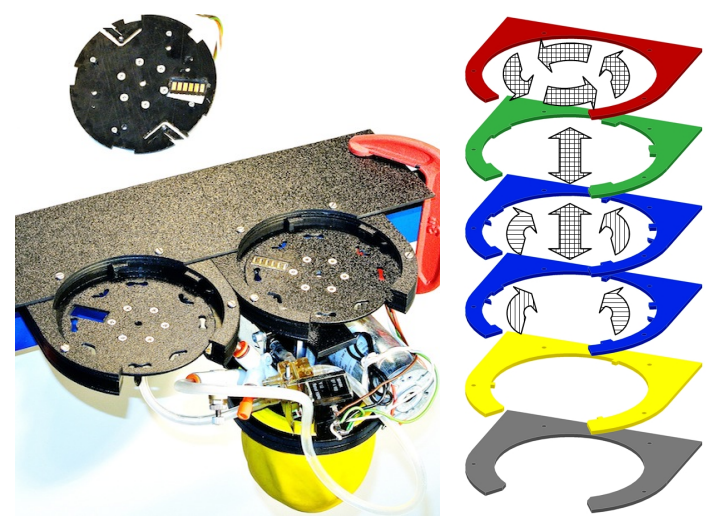

(a) Wrist (top) and two hands (screw driver and jamming gripper) in guides

(b) Quick change end effector exploded

Fig. 12: Quick Change End Effector

and the motions they allow. Arrows hatched with vertical lines are disengage motions and horizontal lines are engage motions. The top layer (red) constrains the robot's arm to move and rotate in the vertical axis only. The next layer (green) contains five teeth to ensure the end effector's orientation is aligned with gaps on the wrist and to ensure the ModLock's screws are aligned with the insertion holes. The teeth are arranged at varying angles from each other: $58^{\circ}, 72^{\circ}, 86^{\circ}, 65^{\circ}, 79^{\circ}$. This is to guarantee no more than one tooth experiences a gap during rotation except when properly aligned. This prevents jamming of the mechanism. Furthermore, each tooth has varied profile dimensions to ensure local contact near each tooth. Two further layers (blue) allow the wrist to rotate and push on the compliant lever to unlock the ModLock. If the wrist is rotated clockwise in the bottom of these two layers, a connection is made; if it is rotated counter-clockwise, a connection is broken. The opposite rotation must be made in the top of these layers to remove the wrist from the guides. The second last layer (yellow) constrains the end effector from rotating relative to PR2's wrist during the connection or disconnection. The last layer (gray) supports the end effector against gravity when the end effector is stored. End effectors are removed through the top or the large gap in the side of the guides once connected to the wrist.

\section{CONCLUSIONS}

A new manual connection mechanism is presented with experimental verification and testing. The aim was to overcome the usual trade off between speed of assembly versus connection strength. We believe this area has received little attention, but is needed to quickly build useful robotic systems.

The ModLock connector enables assembling modules in a short amount of time while still being strong connections. It is capable of supporting the weight of an average person before ultimate tensile failure. It also improves useability by not requiring the user to use nuts and bolts, or other tools to connect modules. This connector has been demonstrated on a variety of modular robot hardware as well as on a manipulator arm functioning as a quick change end effector.

A current downside of the connector is that the faces are not easy to align. Future work can focus on further improving useability by providing guiding features. Additionally, by integrating a power and communications bus into the connection mechanism, we can eliminate the additional step of wiring modules together. In terms of mechanical specifications, mechanical play between modules could be improved. Strength of the mechanism can be increased with larger screws or metal thread inserts into the ABS.

\section{A. Acknowledgments}

The authors thank those who helped with implementation: Stella Latscha, Uriah Baalke, Ian O'hara, Ian Stevens, Jedtsada Laucharoen, and Shai Revzen.

\section{REFERENCES}

[1] A. Castano and A. Behar and P.M. Will. The CONRO modules for reconfigurable robots. Mechatronics, IEEE/ASME Transactions on, 7(4):403-409, Dec. 2002.

[2] Kasper Stoy Andreas Lyder, Ricardo Mendoza. Modular robotics: State of the art. ICRA 2010 Workshop, pages 77-81, 2010.

[3] Modular Robotics Inc. Cubelets website. http://www. modrobotics. com, 2012.

[4] Robotis inc. Bioloid kit, product. http://www.robotis.com/ xe/bioloid_en, 2012.

[5] VEX Robotics inc. Vex Robotics Design System. http://www . vexrobotics.com, 2012.

[6] M.W. Jorgensen, E.H. Ostergaard, and H.H. Lund. Modular ATRON: modules for a self-reconfigurable robot. In Intelligent Robots and Systems, 2004. (IROS 2004). Proceedings. 2004 IEEE/RSJ International Conference on, volume 2, pages 2068 - 2073 vol.2, Sept. - Oct. 2004.

[7] M. Kutzer, M. Moses, C. Brown, M. Armand, D. Scheidt, and G. Chirikjian. Design of a New Independently-Mobile Reconfigurable Modular Robot. IEEE International Conference on Robotics and Automation, May 2010.

[8] R. Moeckel, C. Jaquier, K. Drapel, E. Dittrich, A. Upegui, and A. Ijspeert. Yamor and bluemove an autonomous modular robot with bluetooth interface for exploring adaptive locomotion. In M. O. Tokhi, G. S. Virk, and M. A. Hossain, editors, Climbing and Walking Robots, pages 685-692. Springer Berlin Heidelberg, 2006.

[9] S. Murata, K. Kakomura, and H. Kurokawa. Toward a scalable modular robotic system. Robotics Automation Magazine, IEEE, 14(4):56 -63, Dec. 2007

[10] S. Murata, E Yoshida, K. Tomita, H. Kurokawa, A. Kamimura, and $\mathrm{S}$. Kokaji. Hardware design of modular robotic system. In Proc. of IEEE/RSJ Intl. Conf. on Intelligent Robots and Systems, (IROS), Takamatsu , Japan, 2000.

[11] M. Nilsson. Connectors for self-reconfiguring robots. Mechatronics, IEEE/ASME Transactions on, 7(4):473-474, dec. 2002.

[12] M. Park, S. Chitta, A. Teichman, and M. Yim. Automatic configuration recognition methods in modular robots. The International Journal of Robotics Research, 27(3-4):403-421, 2008.

[13] H.S. Raffle, A.J. Parkes, and H. Ishii. Topobo: a constructive assembly system with kinetic memory. In Proceedings of the SIGCHI conference on Human factors in computing systems, pages 647-654. ACM, 2004

[14] Wei-Min Shen, R. Kovac, and M. Rubenstein. SINGO: A singleend-operative and genderless connector for self-reconfiguration, selfassembly and self-healing. In Robotics and Automation, 2009. ICRA '09. IEEE International Conference on, pages 4253 -4258, May 2009.

[15] P.Wil W-M. Shen. Docking in self-reconfigurable robots. In Proc. of IEEE/RSJ Intl. Conf. on Intelligent Robots and Systems, (IROS), Maui, USA, 2001.

[16] M. Yim. Planetary contingency [education]. Robotics Automation Magazine, IEEE, 15(4):14-16, dec. 2008.

[17] M. Yim, S. Homans, and K. Roufas. Climbing with snake-like robots. In IFAC workshop on mobile robot technology, pages 21-22, 2001.

[18] M Yim, Y Zhang, D Roufas, K Duff, and C Eldershaw. Connecting and disconnecting for chain self-reconfiguration with polybot. Mechatronics, IEEE/ASME Transactions on, 7(4):442-451, Dec. 2002.

[19] Victor Zykov, Phelps Williams, Nicolas Lassabe, and Hod Lipson. Molecubes extended: Diversifying capabilities of open-source modular robotics. 